\title{
Gramsci en Inde : la réception de la pensée dans les «Subaltern
} studies»

\section{Marianna Scarfone}

\section{Citer ce document / Cite this document :}

Scarfone Marianna. Gramsci en Inde : la réception de la pensée dans les «Subaltern studies». In: Raison présente, $\mathrm{n}^{\circ} 175,3 e$ trimestre 2010. Le post-colonial au-delà du post colonial. pp. 41-54;

doi : https://doi.org/10.3406/raipr.2010.4245

https://www.persee.fr/doc/raipr_0033-9075_2010_num_175_1_4245

Fichier pdf généré le 17/03/2019 


\section{GRAMSCI EN INDE : LA RÉCEPTION DE SA PENSÉE DANS LES SUBALTERN STUDIES}

\section{Marianna Scarfone ${ }^{1}$}

\section{Les circonstances académiques et politiques de l'entrée de Gramsci en Inde}

La réception de la pensée de Gramsci dans le monde anglophone commence avec la traduction d'une partie de ses écrits, recueillis dans l'anthologie The modern Prince and other writings ${ }^{2}$ (1957). C'est dans le milieu intellectuel du History Group de Londres et des représentants de la New Left britannique que l'on voit se manifester un premier intérêt international envers la figure de Gramsci. Intellectuels comme Anderson et Hoggart, Hobsbawm et Williams instaurent avec le penseur italien un dialogue fructueux, riche de perspectives et développements différents.

Même la réception indienne de la pensée du philosophe italien pose l'accent - surtout dans un deuxième temps et c'est-àdire avec la naissance et le développement du Collectif des Subaltern Studies - sur l'approche théorique gramscienne qui va bien au-delà du déterminisme lié à l'économique pour s'efforcer d'analyser les superstructures et la dimension culturelle et hégémonique des relations de pouvoir. L'attention des intellectuels indiens se tourne vers la lecture très peu élitiste proposée par Gramsci de la capacité d'élaboration philosophique et d'action pratique de chacun et vers ses réflexions originales autour de la dialectique entre classes dominantes et classes dominées.

Avant de nous concentrer sur le Collectif des Subaltern Studies et sur l'influence que certains concepts gramsciens ont exercé sur celui-ci, il est nécessaire d'esquisser brièvement les étapes de l'entrée de Gramsci dans la culture politique du Sous-continent. Il ne faut pas oublier qu'il s'agit d'une entrée circonscrite à la formation culturelle et à la culture politique d'un cercle étroit et géographiquement situé, et circonscrite à l'univers académique (même si elle est, évidemment, radicale et critique envers la culture dominante), dans le sens que les deux partis communistes, divisés après 
la scission du 1964, l'un lié davantage à l'URSS et l'autre à la Chine, restèrent indifférents aux suggestions du communiste sarde.

Bhabani Sen publie un compte rendu en bengalais de l'anthologie d'écrits gramsciens éditée en 1957 dans Parichay, une revue de la gauche indienne. Mais c'est Susobhan Chandra Sarkar, un historien engagé « qui occupait une position centrale dans la diffusion et la vulgarisation d'idées progressistes parmi les intellectuels ${ }^{3}$, qui le premier analyse et fait circuler et connaître dans les milieux académiques la pensée de Gramsci.

A la fin des années 1950 Sarkar commence à débattre autour de l'œuvre du philosophe italien dans ses cours au Presidency College de Calicut (où Ranajit Guha, initiateur des Subaltern Studies, figure à cette époque parmi les auditeurs); dans la décennie suivante Sarkar poursuit son étude des élaborations gramsciennes, jusqu'à la parution en 1968, dans la revue Mainstream, de l'article "The thought of Gramsci " ${ }^{4}$, pensée qui, dans les mots de Sarkar, " a sans doute enrichi l'arsenal du marxisme et par conséquent mérite aujourd'hui une étude sérieuse et approfondie $»^{5}$.

C'est après la publication de cet article - et grâce à la traduction anglaise d'autres œuvres gramsciennes, de plus en plus intense dans les années 1970 - qu'on voit se consolider la présence de Gramsci dans le débat intellectuel indien.

Dans ce processus de réception, qui se fait donc plus soutenu au cours des années Soixante-dix pour mûrir au cours des années 1980, le rôle joué par le collectif des Subaltern Studies est sans doute significatif.

Ce collectif nait à la fin des années 1970, sur initiative d'un groupe d'historiens basé en partie en Inde et en partie en Angleterre et, guidé, pour ainsi dire, par Ranajit Guha, plus vieux d'une génération par rapport aux autres chercheurs. A partir de 1982, ils publient une série de volumes - jusqu'aujourd'hui douze ont vu le jour - qui portent comme titre Subaltern Studies. Writings on South Asian History and Society.

Si le nom du projet et l'objectif sont éclairés dans la préface au premier volume, il ne faut pas oublier les circonstances politiques concrètes qui conduisent à concevoir un tel projet ceux qui sont les protagonistes et qui vivent en ces années-là à côté de milliers de «subalternes " en révolte.

Comme Guha le déclarera dans sa relation à un congrès qui commémorait la mort de Gramsci en 2007, "la sympathie des 
composantes du collectif allait davantage vers le mouvement paysan qui s'inspirait de la révolution chinoise et des idées de Mao ${ }^{6}$, plutôt que vers la ligne politique des partis communistes indiens ou vers toute narration historico-politique officielle qui, selon un récit univoque, tendait à exclure l'élément paysan en tant que sujet actif de toute action révolutionnaire.

Le mouvement paysan auquel Guha fait référence est le mouvement Naxalite - Naxal étant le district où la première révolte locale contre les propriétaires terriers eut lieu, avant que les émeutes se propagent aussi bien en milieu paysan qu'urbain. Ecrasées violemment par les efforts conjoints du Congrès indien et des deux partis communistes, ces insurrections ont quand même signifié l'émergence d'une opposition critique et d'une action politique visant à la mise en question de la gestion politique postcoloniale.

Comme Guha l'a remarqué, "la force de ce mouvement naît de la désillusion de deux génération à l'égard de la classe politique et des éléments dominants de la société, c'est-à-dire des autorités à tous les niveaux ", en raison des promesses d'un avenir meilleur non tenues. Et c'est de ce même mécontentement intergénérationnel que le collectif des Subaltern Studies tire sa lymphe. Guha tient à souligner ce point pour démontrer que « le projet était une partie organique de la vie réelle de ces années là, qu'il participait au monde auquel il appartenait et qu'il n'était pas tout simplement un point d'observation académique détachée ».

\section{Emprunts gramsciens et réélaborations dans l'historiographie du Sous-continent indien}

Le premier volume de Subaltern Studies, Writings on South Asian History and Society, paraît en $1982^{7}$. Dans sa préface Guha explique le but du projet et ses sources théoriques, ses noyaux thématiques et son approche novatrice à la lecture des archives et à l'écriture de l'histoire, et il suggère les contingences historiques dans lesquelles le projet se situe (qui seront mieux expliquées dans un volume qui paraît en $1988^{8}$ et qui, préfacé par Edward Said, marque le début de la carrière internationale des Subaltern Studies).

Les chercheurs du Collectif articulent des problématiques urgentes de leur présent - celles que la révolte de matrice naxalite avait soulevées - à leur histoire coloniale, ainsi qu'à leur récente histoire nationale, qui jusque-là n'avaient obtenu qu'une attention partielle, une attention qui procédait d'un point d'ob- 
servation élitiste et qui légitimait sans détours la domination colonialiste et la direction nationaliste, dotant a posteriori ces régimes d'un " consensus " de la part de la population que, selon la nouvelle historiographie inaugurée par les Subaltern Studies, elles n'avaient jamais eu.

Pour ces historiens, il s'agit de proposer une réflexion systématique et approfondie sur des thèmes liés à "l'attribut général de la subordination ${ }^{9}$, qui peut se décliner en termes de classe, caste, âge, genre, profession. L'investigation doit traverser " l'histoire, les aspects politiques, économiques et sociaux, les attitudes, l'idéologie et les croyances - voire la culture qui caractérise telle condition ". Leur objectif est de mettre en discussion le corpus officiel d'interprétations relatives au passé et démonter certaines constructions idéologiques canonisées du monde académique et politique, qui placent au centre les groupes dominants dans des historiographies " élitistes ", pour valoriser au contraire le tissu autonome de relations et modalités défini par la politique subalterne.

Ces historiens "marginalisés ", comme ils se définiront plus $\operatorname{tard}^{10}$, puisent des idées et des suggestions de leurs lectures gramsciennes. Ils adaptent à la situation qu'ils veulent analyser la notion de groupes subalternes, celle d'une dialectique entre groupes dominants et subalternes, l'idée d'autonomie, les concepts d'hégémonie et de révolution passive.

\section{Les groupes subalternes et la tâche de l'bistorien intégral}

Avant tout, et comme cela paraît évident, le concept gramscien de " subalterne » ou mieux, de groupes subalternes, est central dans la formulation du projet historiographique des Subaltern Studies. D'un côté ce concept permet de réélaborer « le malaise ressenti par l'ensemble du marxisme extra-occidental à propos d'une définition du sujet révolutionnaire entièrement calquée sur la classe ouvrière métropolitaine "; de l'autre côté, il présente au premier plan la relation dialectique entre dominants et dominés, entre l'élite qui avec tous les moyens aspire à l'hégémonie, et la masse subordonnée - dans le contexte colonial doublement subordonné : à l'autorité indigène traditionnelle et au pouvoir colonialiste - voulant subvertir l'ordre imposé d'en haut.

La référence au penseur italien devient explicite avec la citation, dans la préface au volume du 1982, du " projet en six points 
envisagé par Antonio Gramsci dans ses 'notes sur l'histoire de l'Italie'». " Notes on Italian History » est la section où se trouvent les paragraphes 2 et 5, extrapolés du Cahier 25, dans l'édition partielle anglaise des Cabiers de Prison, publiée en $1971^{11}$ et utilisée dans le monde anglophone jusqu'à la récente édition intégrale anglaise ${ }^{12}$. On en vient ainsi au Cahier 25, intitulé "Aux marges de l'histoire " (Histoire des groupes sociaux subalternes), écrit en 1934. S'il y a déjà des notes sur cet argument, dans le $1^{\text {er }}$ et le $3^{\text {ème }}$ cahiers de prison, écrits en 1930, et dans le Cahier 9, du 1932, c'est seulement avec le cahier " spécial " 25 qu'on trouve une élaboration plus systématique des éléments d'une réflexion originale sur les classes (mot que Gramsci utilise dans une première phase) ou groupes sociaux subalternes (comme il dit dans le cahier susmentionné).

L'étude de l'histoire des classes subalternes, « fonction fragmentée et discontinue de l'histoire da la société civile et, par ce biais, de l'histoire des Etats ", (Q 25, $\mathbb{S} 5^{13}$ ) demande de la part de «l'historien intégral » des critères précis de méthode, que Gramsci distingue justement en six points. Ces-derniers (et les autres points éventuels qui en découlent) reflètent les phases concrètes dans lesquelles ces groupes se développent à partir des stades les plus primitifs de subordination, en passant par des niveaux de plus grande conscience, initiative, organisation, autonomie, jusqu'à une radicale transformation de leur propre position sociale, en arrivant a l'acquisition de l'hégémonie, pour fonder un état " post-subalterne ", comme l'a défini Marcus Green ${ }^{14}$, où il n'y ait pas d'espace pour des relations de domination, exploitation et vexation.

Le fait de reconnaître leur dette envers Gramsci mais de ne pas suivre complètement sa leçon, où la théorie serait finalisée en praxis, et d'avoir tiré du corpus gramscien ce qui les arrangeait, a coûté à ces historiens une critique de leur manière de se référer à la pensée gramscienne. Joseph Buttigieg affirme que « lues hors de leur contexte, ces notes deviennent à peine plus qu'un programme pour une recherche historiographique alternative. Ils ne se rendent pas compte - poursuit-il - que pour Gramsci l'analyse de l'histoire des groupes sociaux subalternes est liée inextricablement à l'articulation d'une stratégie concrète pour un parti politique révolutionnaire $» 15$.

C'est justement en relation à la méthode d'exploitation des sources documentaires que ces chercheurs renvoient à Gramsci et élaborent une stratégie de lecture à plusieurs niveaux des archives de la contre-insurrection. Selon les mots de Gramsci « toute trace 
d'initiative autonome de la part des groupes subalternes devrait être d'une valeur inestimable pour l'historien intégral; il résulte de cela qu'une telle histoire ne peut être traitée que par monographies et que chaque monographie demande une somme considérable de matériaux souvent difficiles à rassembler» $(\mathrm{Q} 25, \mathbb{} 2)$.

\section{L'autonomie et le champ de la politique}

\section{subalterne}

La tâche de "l'historien intégral " est d' " observer et justifier la ligne de développement vers l'autonomie intégrale"

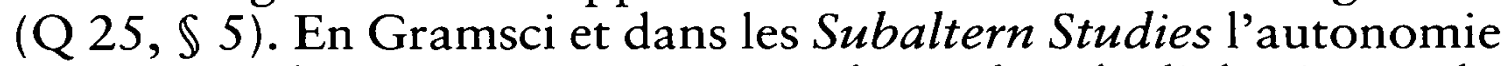
est un mot-clé, une catégorie intermédiaire dans la dialectique subalternité-domination; on peut cependant constater comment les historiens indiens ont tendance à donner à ce mot une acception moins négative que Gramsci. En fait, ils arrivent à donner à ce terme un contenu historique concret, c'est-à-dire qu'ils recouvrent un accomplissement réel, réalisé, de ces formes autonomes d'action politique à niveau de micro-histoire, comme ils suggèrent dans leurs monographies, et pas seulement réalisable dans un avenir incertain, qu'on aurait tendance à étaler sur une échelle qui mesure la transition entre féodalisme et capitalisme et les différents dégrés de modernisation.

Selon Ranajit Guha la spécificité de l'exercice de l'autorité dans les situations coloniales - qu'il qualifie, non sans faire référence à Gramsci, de "domination sans hégémonie »16 - a généré des formes autonomes de politique subalterne que, historiquement articulées dans des mouvements d'opposition et de pensée " autonomes ", la politique des groupes dominants n'a pas su " incorporer » dans ses stratégies hégémoniques.

Les historiens des Subaltern Studies visent à valoriser « la contribution apportée par le peuple - indépendamment de l'élite - au développement du nationalisme ", et donc l'espace autonome de la politique subalterne, dont l'existence n'était pas l'effet de la politique des élites, de l'administration coloniale ou de la mobilisation nationaliste.

David Arnold, un des membres du groupe, soutient que le fort accent mis sur l'autonomie des modes de pensée et d'actions subalternes, désormais mises au centre dans leur « historiographie alternative ", différencient la posture des Subaltern Studies de celle de Gramsci. Les historiens indiens, "tendent à aller plus loin que Gramsci dans l'identification d'un degré supérieur d'autonomie et 
cohésion interne dans la politique paysanne de l'Inde coloniale par rapport a ce que le philosophe italien pouvait voir dans son Italie ". Selon Arnold, Gramsci tend à souligner l'indifférence et la faiblesse interne, l'apoliticité et la passivité de la masse subalterne, «la dispersion et l'isolement de la population rurale $(Q 1, \mathbb{4 4})$, la division entre les paysans mêmes, le manque de lien entre population rurale et urbaine et septentrionale et méridionale - facteurs qui ne peuvent que faciliter le contrôle de la part des classes dominantes.

De plus, dans la perception des historiens des Subaltern Studies ce sont les mêmes formes socioculturelles de l'existence paysanne qui fournissent les éléments vitaux de solidarité à la base des actions collectives. Affaiblissant donc la notion gramscienne d'une culture subalterne dérivée de celle d'élite et conditionnée par celle-ci, ils emphatisent « la capacité des groupes subalternes de formuler une culture compatible avec leurs besoins et leurs expériences plutôt que de reprendre la vision du monde des élites ".

Guha dans son livre publié en 1983, Elementary aspects of peasant insurgency on colonial India ${ }^{17}$, esquisse les caractères principaux récurrents de toute révolte paysanne. Avec un riche spectre d'exemples, aussi ample dans le sens diachronique que géographique, il expose une série d'éléments, de signes et symboles qui affectaient les aspects de la vie paysanne de tous les jours (de la façon de s'habiller aux moyens et codes de communication, de la solidarité communautaire à une ambigüité consciemment cherchée dans les rapports avec les autorités). Ces éléments constituent la spécificité du champ politique des groupes subalternes, desquels ils révèlent l'identité autonome et distincte, développée en opposition, par différence et négation par rapport à celle des groupes dominants.

Visant à doter d'autonomie et d'agency (capacité d'agir) le sujet subalterne, Guha s'éloigne de toute interprétation historique tendant à nier une valeur politique aux révoltes paysannes; sur ce point on constate sa distance par rapport à Hobsbawm et aux historiens marxistes qui, à la recherche d'un paysan porteur d'une rationalité calquée sur le modèle occidental, ne reconnaissent pas les aspects spécifiques d'un sujet qui se constitue, vit et bouge à l'intérieur d'un tissu autonome de relations et modalités d'actions, et tendent à le relier, en tant qu' " arriéré ", à un « espace pré-politique ». L'objectif de Guha et du projet collectif est donc le dépassement d'une vision du subalterne-paysan comme figure du passé. Le propos est de le considérer dans sa réalité historique effective : 
en tant que contemporain du colonialisme et sujet fondamental avec une "conscience ", des pratiques d'action et de résistance qui lui sont propres - de la modernité amorcée par le gouvernement colonial et ses dispositifs de pouvoir.

\section{L'bégémonie en contexte colonial et post- colonial}

De la réflexion de Guha ressort en première ligne le sujet de l'hégémonie. C'est justement la forme spécifique de l'exercice du pouvoir qu'il qualifie de "domination without hegemony "18, qui aurait permis la parution du champ autonome subalterne, articulé à partir de la société civile.

Dans toute relation de pouvoir, la domination et la subordination se conditionnent mutuellement. A l'intérieur de cette relation dialectique, Guha considère nécessaire, dans l'analyse des configurations contingentes de pouvoir, de reconnaître la " composition organique ", c'est-à-dire le poids relatif à chacun des éléments déterminants constituant le couple. Coercition et persuasion seraient les éléments constitutifs de la domination, collaboration et résistance ceux de la subordination. Celui d'hégémonie devient alors un concept dynamique qui représente l'articulation historique concrète du pouvoir, telle que dans la composition organique de la domination la persuasion ait un poids plus grand que la coercition.

Il faut rappeler ici ce que Gramsci visait à saisir avec la notion d' " hégémonie " (qui, en effet, ne diffère pas beaucoup du sens que lui a par la suite donné Guha, si on juxtapose les termes persuasion et consensus-consentement). Dans ses Cabiers de prison, le sens d'hégémonie bascule, sans qu'on puisse aboutir à une définition univoque du terme. Il désigne alternativement une domination exclusive, fondée sur la "force matérielle » et exercée sur le terrain politique, et une forme de " direction plus domination ", qui représenterait le moment du " rapport des forces politiques » où l'on achève "non seulement l'unité des fins économiques et politiques, mais aussi l'unité intellectuelle et morale " $(\mathrm{Q} 13, \mathbb{S} 17)$. En outre «l'exercice normal de l'hégémonie [...] est caractérisé par la combinaison de la force et du consentement qui s'équilibrent de façon variable, sans que la force l'emporte trop sur le consentement, voire en cherchant à obtenir que la force apparaisse appuyée sur le consentement de la majorité » $(Q 13, \mathbb{S} 7)$. 
Guha adapte donc le concept d'hégémonie à la réalité indienne pour conclure qu'en Inde l'élite coloniale et l'élite nationaliste indigène ont en commun de n'avoir pas réussi à créer une hégémonie accomplie qui présuppose, suivant Gramsci, " un consensus actif » $(\mathrm{Q} 6, \mathbb{1} 10)$; et aussi d'avoir constitué ad hoc, avec la complicité de l'historiographie, une " hégémonie illégitime ", qui puisse masquer les moyens violents et répressifs sur lesquels les deux régimes ont fondé leur domination.

Incapable d'articuler ses propres intérêts avec ceux des groupes sociaux impliqués-soumis dans le projet impérialiste, le colonisateur anglais n'a pas su créer une " unité morale et intellectuelle " au delà de l'unité " politique et économique » imposée et un système de significations et valeurs partagées et intériorisées par les masses. Réduisant la politique à une dynamique de "stimulation » du côté impérial et de "réponse » du côté des natifs, l'historiographie colonialiste a produit une vision partielle et idéologique fondée sur l'exclusion arbitraire du contenu de masse de la politique, sur l'effacement du phénomène, qu'on préférait définir «sub-politique ", de la résistance.

La bourgeoisie indienne aurait aussi échoué dans sa tentative de créer une véritable hégémonie à travers laquelle elle pourrait vraiment représenter la société, parler au nom de tout le peuple indien. Le Congrès indien aurait dû élaborer une stratégie de contre-hégémonie à l'égard de la domination anglaise. Comme Gramsci le suggérait à tout groupe social visant à dépasser l'état de soumission, l'élite nationaliste aurait dû " acquérir une hégémonie politique même avant d'être au gouvernement ", parce qu'« un groupe social peut, et même doit, être dirigeant avant de conquérir le pouvoir de gouvernement » $(\mathrm{Q} 1, \mathbb{S} 44)$; en revanche, déjà avant de détenir le pouvoir, elle n'a que partiellement investi le terrain de la politique subalterne et n'a pas su intégrer les intérêts des paysans et des ouvriers dans une hégémonie accomplie. Elle a tenté d'éliminer l'initiative autonome des masses en leur imposant un ordre et une discipline qui ne leur appartenaient pas, en s'appropriant des éléments populaires normalisés utiles à la construction de l'unité nationale.

En niant le postulat libéral-impérialiste d'un consentement à la base de la domination du colonialisme et du nationalisme, Guha et avec lui les autres membres du collectif dévoilent la fausseté des " prétentions hégémoniques " des groupes dominants et la mystification opérée par les narrations d'historiens organiquement liés aux autorités qu'ils se trouvaient côtoyer et soutenir. Ils 
posent donc au cœur de leur réflexion, en tant que "problématique centrale de l'historiographie » indienne contemporaine, " l'étude de l'échec historique de la nation à se créer en tant que telle "19. L'échec dont ils parlent est attribué à "l'inaptitude tant de la bourgeoisie que de la classe ouvrière à déterminer une victoire décisive sur le colonialisme et à achever par la suite une révolution démocratique-bourgeoise ${ }^{20}$.

\section{La transition de l'Etat colonial à l'Etat postcolonial comme révolution passive}

Au lieu d'une révolution de ce genre, il n'y aurait eu qu'une " révolution passive ". Cette dernière notion, empruntée directement à Gramsci, devient dans le langage des historiens des Subaltern Studies " la forme générale de la transition de l'Etat colonial à l'Etat national, post-colonial du $\mathrm{XX}^{\mathrm{c}}$ siècle ${ }^{21}$.

Partha Chatterjee construit son analyse du nationalisme indien dans les différentes étapes de son développement, en interrogeant le rapport avec les formes de savoir-pouvoir occidentales, le rôle de l'élite bourgeoise libérale-progressiste dans la réalité indienne et l'importance des résistances opposées à son projet hégémonique normalisateur de la part de groupes subalternes. Il s'intéresse aux formes spécifiques qui émergent de la disjonction entre l'imaginaire national - construit autour de concepts tels que droit à l'autodétermination, souveraineté populaire et citoyenneté démocratique - et les logiques qui articulent l'expérience vécue par les sujcts, "hétérogène ", pas forcément reproduisant le modèle " modernité-démocratie-nation " d'importation occidentale.

En revenant à Gramsci et à sa définition de révolution passive, on doit faire référence aux cahiers où l'auteur analyse le Risorgimento italien et les raisons de son échec. Dans le cas italien, l'idéologie propre à la bourgeoisie et l'organisation de cette dernière au niveau de choix stratégique et d'architecture hégémonique (dans le double aspect de coercition et de direction morale et intellectuelle), sont restées fragmentaires et incomplètes; de même, la coordination des intérêts bourgeois avec les besoins et les aspirations des masses n'a été que superficielle et sporadique.

La définition récurrente chez Gramsci de révolution passive, la plus générale, et qui devient donc applicable à d'autres contextes, se formule dans les termes suivants : c'est " la nécessité pour la "thèse" de se développer tout entière, jusqu'à réussir à incorporer une partie de l'antithèse elle-même sans se laisser 
"dépasser", c'est-à-dire que, dans l'opposition dialectique, seule la thèse en fait développe toutes ses possibilités de lutte jusqu'à s'accaparer les soi-disant représentants de l'antithèse " $(\mathrm{Q} 15, \mathbb{1 1})$.

La force du concept de révolution passive consiste dans sa nature "métaphorique ", c'est-à-dire dans sa pertinence et sa validité comme "critère d'interprétation " de " toute époque de bouleversements historiques » $(Q 15, \mathbb{S} 62)$, de tous processus de " modifications moléculaires " et de "conservatisme réformiste tempéré » dans lesquels la classe dirigeante s'assure la continuité historique en maintenant une situation cristallisée $(Q 6, \mathbb{1} 162)$ et réduisant la politique au "pouvoir de production et de gouvernement d'un procès de passivité, de standardisation et de broyage ${ }^{22}$.

Celui de révolution passive se révèle ainsi un concept dynamique capable de tracer, en partant de phénomènes historiques précis, un paradigme interprétatif plus général.

La transition de l'état colonial à l'état national postcolonial est analysé par Chatterjee en termes de révolution passive, "la forme politique à travers laquelle la bourgeoisie peut acquérir le pouvoir sans des ruptures retentissantes " $(Q 1, \mathbb{S} 151)$; la forme politique dans laquelle le développement national du capital peut se produire sans résoudre la tension inhérente dans le discours nationaliste qui, voulant se construire comme discours différent par rapport à celui du nationalisme occidental, " reste prisonnier de la structure de pouvoir qu'il entend répudier ${ }^{23}$ et donc n'approfondit pas des formes autonomes d'imagination de la communauté.

La transformation politique voulue par le mouvement nationaliste indien est limitée, "moléculaire ». Il n'a pas la volonté de transformer radicalement les structures du gouvernement anglais. Il ne remet pas directement en question le pouvoir des classes dominantes précapitalistes : il se borne à les neutraliser si nécessaire, à les attaquer de manière sélective et généralement les maintient comme classes alliées, en tant que soutien au nouvel état. Sa stratégie concilie "l'accumulation avec la légitimation, afin d'éviter les rigidités non nécessaires du conflit social $» 24$. Fondée sur l'appropriation de l'action politique des groupes subalternes, cette stratégie produit un modèle de politique nationale où les subalternes sont mobilisés sans pour autant participer : l'Etat national incorpore les classes subalternes dans l'espace imaginaire de la nation, mais il les tient à distance de l'espace politique réel du pouvoir de l'Etat. 
C'est le gandhisme, avec ses aspirations ambigües, qui pose les bases d'une inclusion idéale de l'hétérogénéité de l'expérience subalterne dans la "communauté imaginée » qu'est la nation (B. Anderson), mais qui, en réalité, contribue à son exclusion du procès de développement politique de l'Etat national. En effet, dépouillé de son idiome religieux caractéristique et de sa philosophie anarchique de résistance à l'oppression étatique, le Gandhisme devient lui-même, selon Chatterjee, " une arme idéologique dans les mains de la bourgeoisie indienne " ${ }^{25}$. Déjà Gramsci décrivait le Gandhisme comme une "théorisation naïve et teintée de religion de la révolution passive " $(\mathrm{Q} 15, \mathbb{S} 17)$. C'est probablement cela le point de départ de l'analyse de Chatterjee, qui développe de manière exhaustive l'idée de " traduction » de la catégorie interprétative de la révolution passive dans le contexte indien que Gramsci avait à peine amorcé.

\section{Conclusion}

On a ici esquissé un tableau de l'utilisation et de la réélaboration des concepts gramsciens dans le débat historiographique indien - qui de toute façon ont franchi les confins du Sous-continent et de la discipline dont on parle-des dernières décennies. On a vu comment des expressions et des catégories telles que groupes subalternes, autonomie, hégémonie, révolution passive, ont été reprises et adaptées au contexte indien colonial et post-colonial. Elles se sont révélées comme des clefs précieuses pour interpréter la réalité et la société du Sous-continent, pour saisir les conflits et les contradictions qui en affectent le processus historique. Elles ont contribué à produire une lecture originale, possible grâce à "l'ouverture - dans les mots du fondateur des Subaltern Studies - d'une pensée qui encourage l'adaptation et permet au lecteur une grande liberté d'absorber et de faire de ses idées les siennes ${ }^{26}$.

Dans cette adaptation de la pensée de Gramsci dans des contextes géographiques et chronologiques différents, il apparaît que l'idée de transition - essentielle pour Gramsci, notamment dans sa vision de la nécessité d'un dépassement de la condition de subalternité par les groupes marginalisés en s' " unifiant ", jusqu'à devenir classe hégémonique et donc "Etat » - dans la réception indienne perd sa centralité. Si dans Gramsci on trouve une confiance déterministe dans le chemin suivi par la sulbaternité, à travers différents dégrés d'autonomie, vers la citoyenneté, celle-ci s'estompe dans les Subaltern Studies. L'action, dont on souligne l'autonomie et la politicité, des groupes subalternes ne se décline 
pas nécessairement selon les paradigmes de la " modernité occidentale "; le langage de la citoyenneté et des droits, de la souveraineté populaire et de l'édification nationale n'est pas celui qui parvient nécessairement à mieux décrire les dynamiques de la postcolonialité indienne et la distance entre les politiques imposées d'en haut et les mouvements naissant d'en bas.

1. Doctorante à l'Université de Venise en Histoire Contemporaine. A travaillé d'abord sur la réception indienne de Gramsci, pour ensuite se focaliser sur le rapport entre sciences sociales et colonialisme (Master 2 Paris VII-Bologne). Son intérêt de recherche actuel est la psychiatrie comme dispositif d'hégémonie en contexte colonial.

2. Louis Marks, The Modern Prince and Other Writings, Lawrence and Wishart, London 1957.

3. Barun De, "Susobhan Chandra Sarkar ", dans Id. (dir.), Essays in Honour of Professor S. C. Sarkar, People's Publishing House, Delhi 1976, p. xvii.

4. Susobhan C. Sarkar, "The Thought of Gramsci ", Mainstream, 2 novembre 1968, p. 17-26.

5. Art. cité, p. 26.

6. Ranajit Guha, "Omaggio a un maestro ", dans Gramsci e le culture del mondo, Viella, Roma 2009.

7. Ranajit Guha (dir.), Subaltern Studies I, Oxford University Press, Delhi 1982.

8. Ranajit Guha et Gayatri C. Spivak (dir.), Selected Subaltern Studies, Oxford University Press, New York 1988.

9. Ranajit Guha, "Preface ", dans Id. (dir.), Subaltern Studies I, cité, p. VII.

10. Ranajit Guha, "Introduction ", dans Id. (dir.), A Subaltern Studies Reader I 986-1995, University of Minnesota Press, Minneapolis 1997, p. xiv.

11. Sandro Mezzadra, "Presentazione", dans R. Guha et G. C. Spivak, Modernità e (post) colonialismo, Ombre Corte, Verona 2002, p. 12.

12. Ranajit Guha, "Preface ", dans Id. (dir.), Subaltern Studies I, cité., p. vii. 13. Quintin Hoare et Geoffrey N. Smith (dir.), Selections from Prison Notebooks of Antonio Gramsci, Lawrence and Wishart, London 1971.

14. Antonio Gramsci, Prison Notebooks (sous la dir. de Joseph Buttigieg), Columbia University Press, 1992-2007.

15. Par la lettre $Q$, l'auteur désigne les Quaderni del carcere de Gramsci, présent. Valentino Gerratana, éd. Giulio Eonandi, Turin 197 ((4 vol. XVIII-3369 p.), rééd. Riuniti, 1991, Rome. Les nimbres indiquent le numéro du quaderno et celui du paragraphe (note de l'édition).

16. Marcus E. Green, Gramsci's Method and Analysis of Subaltern Social Groups, paper pour la "Third Conference on Gramscian Studies », 7-10 October 2003, University of Puebla, Mexico, p. 33.

17. Joseph A. Buttigieg, "Sulla categoria gramsciana di subalterni ", Critica Marxista, 1, 1998, p. 58.

18. Cfr. Ranajit Guha, Dominance without Hegemony and its Historiography, dans Id. (dir.), Subaltern Studies VI, Oxford University Press, Delhi 1985, pp. 210-308.

19. Ranajit Guha, On Some Aspects of the Historiography of Colonial India, dans Id. (dir.), Subaltern Studies I, cité, p. 3. 
20. David Arnold, Gramsci and Peasant Subalternity in India, "The Journal of Peasant Studies ", 11, 4, 1984, p. 175.

21. Art. cité, p. 171.

22. Ranajit Guha, Elementary Aspects of Peasant Insurgency in Colonial India, Oxford University Press, Delhi 1983.

23. Ranajit Guha, Dominance without Hegemony, cité.

24. Ranajit Guha, On Some Aspects of the Historiography of Colonial India, cité, p. 5.

25. Ivi.

26. Partha Chatterjee, Nationalist Thought and the Colonial World: A Derivative Discourse?, University of Minnesota Press, Minneapolis 1986, p. 50. 